\title{
Evidencia de validez diagnóstica de la Escala DetectaWeb-Malestar
}

\author{
José A. Piqueras, Mariola García-Olcina, María Rivera-Riquelme y David Pineda \\ Universidad Miguel Hernández de Elche, Elche, España
}

\section{Evidence of diagnostic validity of the DetectaWeb-Distress Scale}

\begin{abstract}
The DetectaWeb-Distress Scale is a web-based scale for screening and detecting internalizing disorder symptoms (anxiety, depression, posttraumatic stress, obsessions and compulsions, and suicidality) in children and adolescents. Previous studies examined the validity and reliability of the scale, however, there are no data on its diagnostic validity. The objective of this study was to examine the evidence of diagnostic validity of the DetectaWeb-Distress scale in a sample of 244 children and adolescents from clinical $(n=51)$ and community $(n=193)$ settings, all of them with the diagnostic process completed. The results indicated that the DetectaWeb-Distress scale is a useful measure from a diagnostic point of view, as it discriminates between people with emotional disorders such as anxiety and depressive disorders and suicidality, and those without, presenting ROC values of approximately .80 and good sensitivity and specificity for detecting the main emotional disorders. The DetectaWeb-Distress Scale is a valid measure and diagnostically useful for detecting and identifying children and adolescents with anxiety disorders, depression and suicidality, with the advantage that it is a short measure, specifically developed for Internet use, especially relevant in the era of COVID-19.
\end{abstract}

Keywords: DetectaWeb-Distress; assessment, anxiety, depression, children, adolescents.

Resumen: La Escala DetectaWeb-Malestar es una escala de detección del malestar emocional a través de internet que evalúa síntomas de trastornos interiorizados (ansiedad, depresión, estrés postraumático, obsesiones y compulsiones y suicidalidad) en niños y adolescentes. Estudios anteriores han examinado la validez y fiabilidad de la escala, sin embargo, no cuenta con datos relativos a su validez diagnóstica. El objetivo de este estudio es examinar las evidencias de validez diagnóstica de la escala DetectaWeb-Malestar en una muestra de 244 niños y adolescentes procedentes de contextos clínico $(n=51)$ y comunitario $(n=193)$, todos con un proceso diagnóstico completado. Los resultados indicaron que la escala DetectaWeb-Malestar es una medida útil desde el punto de vista diagnóstico, ya que discrimina entre personas con trastornos de emocionales, como son los de ansiedad, depresión y suicidalidad y los que no lo padecen, presentando valores ROC en torno a .80 y adecuada sensibilidad y especificidad para detectar los principales trastornos emocionales. La escala DetectaWeb-Malestar es una medida válida y útil desde el punto de vista diagnóstico para detectar e identificar niños y adolescentes con problemas de ansiedad, depresión y suicidalidad, con la ventaja de que es una medida breve y desarrollada específicamente para su uso a través de Internet, especialmente relevante en la era de la COVID-19.

Palabras clave: DetectaWeb-Malestar; evaluación; ansiedad; depresión; niños y adolescentes.

\section{Introducción}

La depresión y la ansiedad son una de las causas más frecuentes de enfermedad y discapacidad en niños y

Recibido: 26 de octubre de 2020; aceptado: 11 de noviembre de 2020 Correspondencia: David Pineda, Departamento de Psicología de la Salud, Universidad Miguel Hernández de Elche, Avda. de la Universidad, s/n. Edificio Altamira 03202 Elche, España. Correo-e: dpineda@umh.es Agradecimientos: Este estudio ha sido financiado por el Vicerrectorado de Investigación y Desarrollo Tecnológico de la Universidad Mi- adolescentes entre 10 y 19 años, estando la depresión en el primer puesto y la ansiedad en el octavo. Así mismo, el suicidio constituye también un tema de especial relevancia (Sánchez-Teruel, Muela-Martínez y García-León

guel Hernández (BANCAJA-UMH, convocatoria 2010), el Departamento de Atención a la Salud (Consejería de Sanidad) de la Comunidad Valenciana (SMI 10/2014) y una beca para la contratación de personal investigador de carácter predoctoral concedida a la tercera autora (ACIF/2015/155; Programa VALi+d; Consejería de Educación, Investigación, Cultura y Deporte de la Comunidad Valenciana). 
(2018), el cual se encuentra entre las tres causas principales de mortalidad entre los adolescentes (World Health Organization, 2014). Más concretamente, un estudio reciente de revisión meta-analítica sitúa las tasas de prevalencia mundial de estos trastornos en un $6.5 \%$ para los trastornos de ansiedad y $2.6 \%$ para los trastornos depresivos en población infantojuvenil con una marcada comorbilidad entre ambos trastornos (Al-Asadi, Klein y Meyer, 2015; Cummings, Caporino y Kendall, 2014; de Ross, Gullone y Chorpita, 2002; Melton, Croarkin, Strawn y McClintock, 2016). Por su parte, su prevalencia en muestras clínicas varía entre el $13 \%$ y el $41.9 \%$ para la ansiedad y entre el $13 \%$ y el $16.9 \%$ para depresión (Walter et al., 2016).

Por lo tanto, es necesario y beneficioso para la práctica clínica la evaluación de ambos trastornos y síntomas de depresión o ansiedad en un niño o adolescente a la hora de realizar un primer diagnóstico o en la detección de estos síntomas en poblaciones generales como las escolares (Alcántara-Jiménez y Garcia-Lopez, 2017; Ebesutani et al., 2010; Moran, Cupani, Azpilicueta, Piqueras y Garcia-Lopez, 2019).

Sin embargo, cualquier aproximación a la evaluación de estos trastornos conlleva el problema de la falta de tiempo del profesional de la salud mental para realizar una evaluación diagnóstica de cada uno de los trastornos. Por ello la práctica más común es el empleo de pruebas de autoinforme para el screening o cribado de estos síntomas (Ebesutani et al., 2012).

Los instrumentos de autoinforme más utilizados para evaluar la ansiedad en niños y adolescentes han sido la clásica Escala Multidimensional de Ansiedad para Niños (MASC; García-Villamisar, Vidal y Yenes, 2002; March, Parker, Sullivan, Stallings y Conners, 1997) y la Escala de Ansiedad Manifiesta en Niños-Revisada (CMAS-R; Reynolds y Richmond, 1997), la Spence Children's Anxiety Scale (SCAS; Hernández Guzmán et al., 2010; Orgilés, Méndez, Spence, Huedo-Medina y Espada, 2012; Spence, 1997), y más recientemente, Muris y colaboradores han desarrollado the Youth Anxiety Measure for DSM-5 (YAM-5; Muris et al., 2017), una nueva escala para evaluar los trastornos de ansiedad en niños y adolescentes siguiendo criterios DSM-5 que miden diferente tipos de ansiedad. Por otro lado, el Children's Depression Inventory (CDI; Kovacs, 1992), (Kovacs, 1992) ha sido uno de los instrumentos más utilizados para la evaluación de la depresión. Son aún más escasos los instrumentos que miden ansiedad y depresión de forma conjunta como la Revised Child Anxiety and Depression Scale (RCADS; Chorpita, Yim, Moffitt, Umemoto y Francis, 2000; Sandín, Valiente y Chorot, 2009) y la RCADS-30 (Sandín, Chorot, Valiente y Chorpita, 2010).
Utilizándose como método de screening para la detección de síntomas compatibles con trastornos de ansiedad y depresión (Chorpita, Moffitt y Gray, 2005; Pineda, Martín-Vivar, Sandín y Piqueras, 2018).

Sin embargo, ninguna de estas medidas ha sido desarrolladas para ser aplicadas directamente a través de internet. Tampoco permiten evaluar un amplio rango de los síntomas de ansiedad, depresión, suicidalidad, estrés postraumático y trastorno obsesivo-compulsivo, ni se han desarrollado en el marco de un programa de detección de los problemas emocionales.

En consecuencia, considerando lo anterior, nuestro equipo creó una nueva medida, la Escala DetectaWeb-Malestar, el primer cuestionario de evaluación basado en una web para la evaluación de subtipos específicos de ansiedad y algunos de los trastornos emocionales relacionados con la ansiedad más comunes, como la depresión, el trastorno obsesivo-compulsivo, el trastorno de estrés postraumático, el suicidio y el malestar general. Hasta el momento el estudio sobre el proceso de creación de la medida y estudio piloto con una muestra de adolescentes de entre 14 y 18 años proporcionó el apoyo inicial para la fiabilidad y validez de la DetectaWeb-Malestar (García-Olcina, Piqueras y Martinez-González, 2014). La medida tuvo una buena consistencia interna para la Escala Global de Malestar $(\alpha=.87)$ y correlaciones adecuadas con medidas relacionadas de ansiedad y depresión con la RCADS-30 ( $r=.40-87)$. Además, otro estudio demostró que, de forma preliminar, esta medida es una herramienta fiable, válida y útil para evaluar los trastornos emocionales en una muestra clínica (García-Olcina et al., 2017) y un trabajo publicado recientemente que, empleando una muestra de 1.499 participantes (de 8 a 18 años de edad), halló apoyo para el modelo estructural de 10 factores correlacionados, invarianza en función de la edad y el sexo, adecuada consistencia interna y diversas evidencias de validez, así como diferencias de género y edad en algunas de las puntuaciones, concluyendo que la DetectaWeb-Malestar es una herramienta online válida, innovadora y útil para la detección y evaluación de programas de intervención para la salud mental y el bienestar en niños y adolescentes (Piqueras, García-Olcina, Rivera-Riquelme, Martínez-González y Cuijpers, 2020). Sin embargo, no hay ningún estudio que haya examinado la validez o utilidad diagnóstica de la escala.

Por ello, el objetivo de este trabajo fue examinar la precisión diagnóstica de DetectaWeb-Malestar mediante el análisis de la capacidad del DetectaWeb-Malestar para discriminar entre muestras con diagnóstico de los problemas emocionales: malestar emocional (ansiedad, depresión y suicidalidad) y sin él; y mediante el cálculo de análisis de las curvas ROC, con los 
consiguientes puntos de corte basados en el mejor equilibrio entre sensibilidad y especificidad de las escalas del cuestionario.

\section{Método}

\section{Participantes}

La muestra general consistió en 243 niños y adolescentes, de los que 193 procedían de contexto escolar (79.40\%), y 50 de contexto clínico $(20.60 \%)$ (véase la Tabla 1). Del total de la muestra, 138 (56.80\%) fueron varones y la edad media fue $11.51(D T=2.68 ;$ rango $=$ 8-18 años). La distribución por sexo de ambas muestras no fue homogénea $\left(\chi_{(1,243)}^{2}=7.60 ; p=.006\right)$, encontrado un mayor número de chicos en la muestra clínica. Res- pecto a la edad de los participantes, no se hallaron diferencias estadísticamente significativas entre los grupos $\left(t_{(241)}=-0.81, p=.42\right)$.

El nivel socioeconómico de la muestra fue mayoritariamente medio $(n=80,33.60 \%)$ y medio-bajo $(n=$ $144,60.50 \%)$ y la nacionalidad española mayoritaria ( $n$ $=224 ; 94.10 \%$ ). No se hallaron diferencias entre los grupos en lo relativo a la distribución por nivel socioeconómico $\left(\chi_{(2,243)}^{2}=3.60, p=.16\right)$ ni por país de origen $\left(\chi^{2}\right.$ $\left.{ }_{(1,243)}=0.36 ; p=.55\right)$.

La distribución en base al sexo, edad, nacionalidad, y nacionalidad para cada grupo fue la que se observa en la Tabla 1.

Respecto a la muestra clínica, los diagnósticos por parte de los profesionales de la salud mental fueron los que aparecen en la Tabla 2.

Tabla 1. Variables sociodemográficas

\begin{tabular}{|c|c|c|}
\hline & $\begin{array}{c}\text { Clínica } \\
(n=50)\end{array}$ & $\begin{array}{c}\text { Escolar } \\
(n=193)\end{array}$ \\
\hline $\operatorname{Edad}[\operatorname{Media}(D . T)]$. & $11.78(2.82)$ & $11.44(2.65)$ \\
\hline \multicolumn{3}{|l|}{$\operatorname{Sexo}[n(\%)]$} \\
\hline Mujeres & $13(26.00)$ & $92(47.70)$ \\
\hline Hombres & $37(74.00)$ & $101(52.30)$ \\
\hline \multicolumn{3}{|l|}{ Nivel socioeconómico (FAS) $[n(\%)]$} \\
\hline Bajo & $24(48.00)$ & $121(62.70)$ \\
\hline Medio & $22(44.00)$ & $62(32.10)$ \\
\hline Alto & $4(8.00)$ & $10(5.20)$ \\
\hline \multicolumn{3}{|l|}{ Nacionalidad $[n(\%)]$} \\
\hline Española & $48(96.00)$ & $181(93.80)$ \\
\hline Otras & $2(4.00)$ & $12(6.20)$ \\
\hline \multicolumn{3}{|l|}{ Procedencia $[n(\%)]$} \\
\hline \multicolumn{3}{|l|}{ Clínica } \\
\hline USMI-A del área 18 (San Vicente del Raspeig) & $30(60.00)$ & - \\
\hline USMI-A del área 19 (Elche-El Raval) & $16(32.00)$ & - \\
\hline Unidad de Psicología Forense del CPA de la UMH & $4(8.00)$ & - \\
\hline \multicolumn{3}{|l|}{ Comunitaria/escolar* } \\
\hline Elche & - & $51(26.40)$ \\
\hline San Juan de Alicante & - & $25(13.00)$ \\
\hline Elda & - & $61(31.60)$ \\
\hline Orihuela & - & $17(8.80)$ \\
\hline Novelda & - & $12(6.20)$ \\
\hline Alcoi & - & $27(14.0)$ \\
\hline
\end{tabular}

Nota. FAS: Family Affluence Scale. USMI-A: Unidad de Salud Mental Infantil y de la Adolescencia; CPA: Centro de Psicología Aplicada. 


\section{Instrumentos}

Escala DetectaWeb-Malestar (García-Olcina et al., 2014). Este es un cuestionario de detección vía web para los trastornos mentales emocionales en niños y adolescentes, que fue creado en el año 2014 (Olcina et al., 2014). Consta de 30 ítems que evalúan los síntomas de los trastornos de ansiedad en general y más específicamente, el trastorno ansiedad de separación (TAS), la fobia específica (FE), la fobia social (FS), el trastrono de ansiedad generalizada (TAG), el trastorno de pánico con agorafobia ( $\mathrm{Tp} / \mathrm{Ag})$, el trastorno de estrés postraumático (TEPT) y el trastorno obsesivo-compulsivo (TOC); así como los trastornos depresivos unipolares como son la depresión mayor y el trastorno distímico (DM y TD); además también explora las tendencias suicidas (ideación, planes y tentativas). Sigue un formato de respuesta tipo Likert $(0=\langle$ nunca», $1=\langle$ a veces», $2=\langle$ a menudo» y $3=$ «siempre»). Estudios previos ha brindado apoyo inicial para la fiabilidad y validez de la escala de DetectaWeb-Malestar evaluando ansiedad, depresión y suicidalidad en niños y adolescentes, tanto en población comunitaria/escolar (Olcina et al., 2014; Piqueras et al., 2020) como clínica (García-Olcina et al., 2017). La fiabilidad de la puntuación total con la presente muestra ha sido de .91.

Tabla 2. Distribución de frecuencias de diagnósticos clínicos de los participantes de la muestra clínica $(n=50)$.

\begin{tabular}{|c|c|}
\hline Diagnóstico* & Muestra clínica \\
\hline Problemas clínicos sin diagnóstico específico & $4(8 \%)$ \\
\hline Trastornos adaptativos & $3(6 \%)$ \\
\hline Trastorno de estrés postraumático & $1(2 \%)$ \\
\hline Trastornos de relación & $1(2 \%)$ \\
\hline Trastornos de ansiedad & $5(10 \%)$ \\
\hline Trastornos depresivos unipolares & $0(0 \%)$ \\
\hline Trastornos afectivos & $1(2 \%)$ \\
\hline Trastorno obsesivo-compulsivo & $1(2 \%)$ \\
\hline Trastorno por Déficit de Atención/Hiperactividad & $10(20 \%)$ \\
\hline Trastorno de la conducta & $8(16 \%)$ \\
\hline Trastorno por Déficit de atención/Hiperactividad y Trastorno de Conducta & $6(12 \%)$ \\
\hline Trastorno por TICS & $1(2 \%)$ \\
\hline Trastornos generalizados del desarrollos o espectro autista & $1(2 \%)$ \\
\hline Trastornos de la conducta alimentaria & $2(4 \%)$ \\
\hline Problemas de sobrepeso/obsesidad & $2(4 \%)$ \\
\hline Trastornos de la identidad sexual & $2(4 \%)$ \\
\hline Trastornos de control de esfínteres & $1(2 \%)$ \\
\hline Discapacidad intelectual leve & $1(2 \%)$ \\
\hline Total & $50(100 \%)$ \\
\hline
\end{tabular}

Nota. $*$ = Diagnósticos a través del juicio clínico de los profesionales de la salud mental de las USMIS (criterios CIE-10).

Family Affluence Scale (FAS; Boyce, Torsheim, Currie y Zambon, 2006; Currie, Elton, Todd y Platt, 1997). La FAS evalúa el estatus socioeconómico mediante 4 preguntas referidas a ciertas posesiones de las familias, tales como coche, ordenador, habitación propia y viajes de vacaciones familiares. Las puntuaciones pueden clasificarse en tres categorías de riqueza familiar: nivel bajo, medio y alto. Ha mostrado validez de criterio y de constructo adecuada en estudios previos con adolescentes (Boyce et al., 2006).

Escala Revisada de Depresión y Ansiedad Infantil (RCADS-30; Sandin et al., 2010). Se trata de una versión abreviada de 30 ítems del RCADS (Chorpita et al., 2000). Esta escala evalúa los problemas de ansiedad y depresión en niños y adolescentes, presenta buenas propiedades psicométricas y está formada por 30 ítems con 
6 subescalas que corresponden a TAS, FS, TAG, Tp, TOC y DM. La escala se puntúa de 0 a 3 (nunca, a veces, a menudo y siempre). Una revisión reciente señala las excelentes propiedades psicométricas de esta versión, equivalente a la original (Piqueras, Martín-Vivar, Sandin, San Luis y Pineda, 2017), una estructura factorial equivalente en muestras clínicas y comunitarias, así como puntos de corte óptimos para detectar problemas emocionales (Piqueras, Pineda, Martín-Vivar y Sandin, 2017). La fiabilidad obtenida con la presente muestra ha sido de .93 .

Subescala de Fobia Especifica del Spence Children's Axiety Scale (SCAS; Orgilés et al., 2012). Para nuestro estudio utilizamos la subescala de fobia específica formada por cinco ítems con 4 alternativas de respuesta tipo Likert $(0=$ Nunca, $1=A$ veces, $2=A$ menudo, $3=$ Siempre), con una consistencia interna promedio de una revisión de 32 estudios de la SCAS de .64 (Orgilés, Fernández-Martínez, Guillén-Riquelme, Espada y Essau, 2016), presentando el mismo valor de consistecnia en nuestro estudio.

Children's Revised Impact of Event Scale (CRIES; Perrin, Meiser-Stedman y Smith, 2005). Es una escala de detección que se utiliza para medir el Trastorno por estrés post traumático en niños a partir de 8 años. Está formada por 8 ítems con 4 alternativas de respuesta tipo Likert $(0=$ Nunca, $1=$ Rara vez, $3=$ A veces, $5=$ Frecuentemente). La consistencia interna de la escala ha sido de .93 .

Cuestionario de Fortalezas y Debilidades (Strengths and Difficulties Questionnaire; SDQ; Goodman, 1997). El SDQ es ampliamente utilizado para la evaluación de diferentes problemas emocionales y conductuales en niños y adolescentes. Consta de 25 afirmaciones distribuidas en cinco subescalas: síntomas emocionales, problemas de conducta, hiperactividad, problemas de relación con los compañeros y conducta prosocial. En este estudio utilizamos la versión española de la SDQ autoinformado de 1117-años que incluye el suplemento de impacto (www.sdqinfo.org). Esta versión utiliza el formato de respuesta tipo Likert de tres opciones; por lo tanto, la puntuación en cada subescala varió de 0 a 10. Las cuatro primeras subescalas ofrecen una puntuación total de dificultades. El SDQ ha mostrado buenas propiedades psicométricas en población española (alfas de Cronbach entre 69 y .78) (Ortuño-Sierra, Fonseca-Pedrero, Paino, Sastre i Riba y Muñiz, 2015). La consistencia interna de la prueba con nuestra muestra ha sido de .85 para la escala total.

Inventario de Salud Mental-5 (Mental Health Inventory-5; MHI-5; Berwick et al., 1991). El MHI-5 es una versión corta del MHI (38 ítems) (Veit \& Ware, 1983). El MHI-5 también fue desarrollado para uso en la pobla- ción general, incluyendo ítems para evaluar el bienestar psicológico. Este inventario consta de cinco reactivos sobre el estado de ánimo durante el mes pasado, que miden la presencia de bienestar psicológico ( 2 preguntas) y la ausencia de malestar (3 ítems invertidos). MHI-5 utiliza un sistema de respuesta de 6 puntos. En nuestro estudio de adaptación, el formato de respuesta ha sido adaptado a un formato tipo 4-punto de Likert $(0=$ nunca, $1=$ algunas veces, $2=$ a menudo y $3=$ siempre). Una puntuación mayor indica mejor salud mental. El alfa de Cronbach obtenido con la presente ha sido de .74.

World Health Organization-5; Well-being Index (WHO-5; World Health Organization, 1998). Es una escala breve y genérica para medir el nivel de bienestar subjetivo, destinado a población infantil y adulta. Los 5 elementos del WHO-5 evalúan aspectos tales como estado de ánimo positivo, calma y relajación, actividad/firmeza y el interés general, entre otros. El cuestionario emplea un formato de respuesta que va desde el 5 (todo el tiempo) al 0 (nunca). Una mayor puntuación indica un mayor bienestar y presenta una consistencia interna de .89. La consistencia en nuestro estudio ha sido de .86 .

The Anxiety Disorders Interview Schedule for DSMIV: Child Version (ADIS-IV-C; Silverman, Albano y Sandín, 2003). Es la adaptación para niños de 6 a 18 años de la entrevista de Brown y colaboradores (Brown, Di Nardo y Barlow, 1994) para el diagnóstico de los trastornos de ansiedad en niños y adolescentes según el DSM-IV. Aunque está diseñada para evaluar los trastornos de ansiedad, no obstante también evalúa el diagnóstico de trastornos del estado de ánimo, trastornos externalizantes, además permite realizar un screening sobre el abuso de sustancias, la esquizofrenia, el mutismo selectivo, los trastornos de la conducta alimentaria y los trastornos somatoformes. También permite obtener información relevante sobre la conducta de rechazo escolar, relaciones interpersonales, áreas específicas de interferencia y estresores. Para nuestro estudio utilizaremos la versión española para niños de Silverman et al. (2003), que presenta buenas propiedades psicométricas. Empleamos únicamente el bloque de la entrevista que evalúa los trastornos de ansiedad (ansiedad por separación, fobia social, fobia específica, ansiedad generalizada, trastorno obsesivo compulsivo, estrés post traumático) y del estado de ánimo (depresión mayor y distimia). Estudios previos han indicado que muestra una adecuada fiabilidad test-retest para todos los trastornos evaluados $(r$ $=.75-.92$ ) (Silverman et al., 2003). En nuestro estudio, el $20 \%$ de las entrevistas fueron grabadas con la intención de analizar el acuerdo interjueces. La fiabilidad entre evaluadores para los diagnósticos de ansiedad y depresión fue excelente $(\kappa=.90)$. 


\section{Procedimiento}

Se trata de un estudio transversal, observacional y multicéntrico con niños y adolescentes de centros clínicos y escolares de primaria y secundaria (véase Tabla 1). Los participantes fueron evaluados entre 2014 y 2017 mediante el programa online DetectaWeb, posteriormente se les realizó una entrevista clínica diagnóstica por profesionales formados en el manejo del ADIS-IV-C. Cabe subrayar que los participantes de la muestra escolar fueron una submuestra formada por 193 participantes elegidos al azar de una muestra utilizada en otro estudio (Piqueras et al., 2020). El estudio fue aprobado por el comité de investigación y ética de la Universidad Miguel Hernández de Elche. Todos los padres de los participantes firmaron el consentimiento informado para su participación en el estudio. Además, todas las escuelas y centros clínicos participaron voluntariamente en este estudio previa obtención de la autorización correspondiente tanto del departamento de psicología, en caso de los colegios, como de los gerentes en caso de los centros clínicos. Una descripción exhaustiva del procedimiento, las características de la plataforma online, las cuestiones éticas, etc., pueden consultarse en un artículo reciente que describe el protocolo completo del estudio DetectaWeb (Piqueras, Garcia-Olcina, Rivera-Riquelme, Rodríguez-Jiménez, Martínez-González y Cuijpers, 2017).

\section{Análisis estadísticos}

Los análisis se llevaron a cabo con los programas estadísticos SPSS 24 y el programa estadístico EQS 6.3. En un primer paso, el análisis de la fiabilidad total y de cada subescala que se calculó mediante el coeficiente alfa de Chronbach basado en la matriz de correlaciones policóricas y el coeficiente omega de McDonald (McDonald, 1999) ya que se considera un estimador mayor de la fiabilidad que el alfa de Cronbach (Dunn, Baguley y Brunsden, 2014). En segundo lugar, se puso a prueba el ajuste del modelo de diez factores correlacionados propuesto por los autores (Piqueras et al., 2018), así como el modelo factorial basado en el DSM 5, en el que estos diez factores cargan a su vez en dos factores de segundo orden y estos dos a su vez en un factor general de tercer orden (American Psychiatric Association, 2013). Para ello empleamos el método de máxima verosimilitud robusta (MLR, Maximum Likelihood Robust). Para el análisis del ajuste se utilizaron los índices Chi-cuadrado $\chi^{2}$, Chi-cuadrado $\chi^{2}$ entre grados de libertad $\left(\chi^{2} / \mathrm{gl}\right.$; Chau, 1997), El Root Mean Square Error of Aproximation (RMSEA; Browne \& Cudeck, 1993; Schumacker \& Lomax, 2004) the Comparative Fit Index (CFI; Bentler,
1990), Standardized Root Mean Square Residual (SRMR), El Akaike Information Criterion (AIC), el Goodness of Fit Index (GFI) y el Adjusted Goodness of Fit Index (AGFI; Jöreskog \& Sörbom, 1993).

En tercer lugar se calculó, la validez convergente-discriminante mediante los coeficientes de correlación entre las puntuaciones de DetetctaWeb y RCADS-30 y medidas de bienestar y malestar psicológico, utilizamos los criterios de Cohen para evaluar los tamaños del efecto (TE) de las correlaciones (Cohen, 1988; Lipsey y Wilson, 2001). En cuarto lugar, se aportan comparaciones entre los grupos de procedencia clínica y comunitaria con y sin diagnóstico en las escalas de DetectaWeb-Malestar. Estimamos también la diferencia media estandarizada $(d$ de Cohen) para aportar la magnitud de las diferencias (Cohen, 1988). Con objeto de establecer la capacidad de las subescalas de DetectaWeb-Malestar para discriminar entre sujetos clínicos y no clínicos, se realizaron los análisis de las curvas ROC (Receiver Operation Characteristic) en base a los diagnósticos derivados de la entrevista diagnóstica semiestructurada ADIS-IV-C. Seguimos la clasificación descrita por Metz (Metz, 1978), según la cual la precisión de una medida diagnóstica se centra en la curva ROC: $.90-1.00=$ excelente, $.80-.90=$ buena, $.70-.80$ $=$ adecuada, $.60-.70=$ pobre, $<.60=$ mala. Por último, se obtuvieron los puntos de corte recomendados a partir del cálculo de los índices de sensibilidad y especificidad, así como del índice de Youden (Youden, 1950), que nos orienta a cerca de cual es el punto de corte que maximiza la precisión de las clasificaciones.

\section{Resultados}

\section{Análisis factorial confirmatorio}

Se puso a prueba el modelo de estructura factorial de diez factores correlacionados, que cuenta con 3 ítems por factor y el modelo factorial basado en el DSM 5 hallado por Piqueras y cols. (Piqueras et al., 2018). En la Tabla 3 se muestran los valores de ajuste de los modelos planteados. El modelo A que corresponde al modelo de 10 factores correlacionados es el que presenta mejores índices de ajuste, no obstante, el modelo B basado en el DSM 5 también presenta ajustes adecuados. La Tabla 4 muestra los pesos estandarizados de cada ítem sobre el factor al que pertenecen que están por encima de $.60(.60-.90)$.

\section{Fiabilidad}

La fiabilidad fue calculada mediante el alfa de Cronbach basados en la matriz de correlaciones policóricas, siendo para la puntación total de .91 (véase Tabla 3). Las 
Tabla 3. Índices de Bondad de ajuste del análisis factorial confirmatorio

\begin{tabular}{|c|c|c|c|c|c|c|c|c|c|}
\hline Modelo & S-B $\chi^{2}$ & $g l$ & $\chi^{2} / \mathrm{gl}$ & $\begin{array}{c}\text { RMSEA } \\
\text { (I.C. } 90 \%)\end{array}$ & SRMR & CFI & GFI & AGFI & AIC \\
\hline Modelo A & 451.28 & 360 & 1.25 & $\begin{array}{c}.03 \\
(.02-.04)\end{array}$ & .06 & .93 & .85 & .81 & -268.71 \\
\hline Modelo B & 518.32 & 392 & 1.32 & $\begin{array}{c}.03 \\
(.03-.04)\end{array}$ & .07 & .90 & .83 & .80 & -265.68 \\
\hline
\end{tabular}

Nota. Modelo A: modelo 10 factores correlacionados; Modelo B: modelo basado en el DSM5 con 10 factores de primer orden más 2

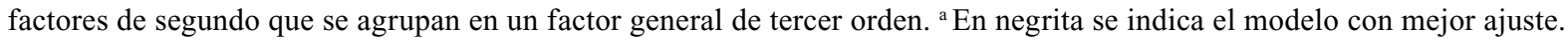

consistencias de las subescalas se encontraron entre .55 (TOC) y .84 (S). Los resultados con los cálculos de los coeficientes omega de McDonald fueron ligeramente inferiores $(.77-.91)$, aunque sin grandes cambios.

\section{Validez convergente-divergente}

Se analizó la validez convergente mediante el coeficiente de Pearson entre las subescalas y puntuaciones totales del DetectaWeb-Malestar y RCADS-30 y otras medidas de malestar y de bienestar (véase Tabla 5). Las subescalas del DetectaWeb-Malestar obtuvieron correlaciones significativas con escalas las homólogas de la RCADS-30, con valores de $r$ entre .60 y .85 , que se corresponde con tamaños del efecto grandes. Para las escalas no homólogas con el DetectaWeb-Distress se obtuvieron valores entre .50 y .65 con otras escalas. También se hallaron relaciones negativas significativas del DetectaWeb-Malestar con medidas de salud mental (véase Tabla 5).

\section{Diferencias en DetectaWeb-Malestar entre el grupo de procedencia clínica y comunitaria}

Como se observa en la Tabla 6, las diferencias fueron significativas en todas las subescalas. Además, los tamaños del efecto fueron grandes en todas las comparaciones excepto en la realizada para la subescala de fobia social, donde solo se halló una magnitud de las diferencias mediana.

\section{Sensibilidad y especificidad}

El área bajo la curva (AUC) para la puntuación total de la Escala DetectaWeb-Malestar fue de $.80(p<.01$; intervalo de confianza del $95 \%=.72$ a .88 ), lo que sugiere que hay un $80 \%$ de probabilidad de que un niño o adolescente con sintomatología alta o baja en malestar emocional sea bien clasificado como participante con este problema o sin él, respectivamente. En la Tabla 8, se muestran las AUC de las subescalas, así se observa como el AUC de DM fue de .91, esto significa que hay un $91 \%$ de probabilidad de que el sujeto puntúe alto en esta subescala. Referente a las demás subescalas (Tabla 7), la D presentó una AUC de .79, el S de .71; referente a las subescalas de ansiedad el AUC del TAS fue de .80, para la FS y FE fue de .81 y .79 respectivamente, para el Tp/Ag de .78. Para el TAG de .77 y para el TOC y el TEPT fue de .91 y .72 respectivamente. En el caso de S y TEPT, presentan una precisión diagnóstica baja, como se puede ver en la Tabla 7, los intervalos inferiores son menores a .40. Respecto a las diferentes puntuaciones que se pueden obtener con la Escala DetectaWeb-Malestar, encontramos un AUC de .79 para los trastornos depresivos, de .78 para los trastornos de ansiedad. De .80 para ambos trastornos y de .79 para cualquier trastorno. En la Tabla 7, se muestra la sensibilidad y especificidad de las puntuaciones de las subescalas del DetectaWeb-Malestar para la detección de trastornos específicos, diagnosticados con la ADIS-IV-C. Se seleccionaron puntos de corte para proporcionar una mejor sensibilidad y especificidad para cada medida.

Respecto a las diferentes puntuaciones que se pueden obtener con la Escala DetectaWeb-Malestar (Tabla 8), encontramos que tanto para los trastornos depresivos, como para los trastornos de ansiedad, presentar cualquiera de estos trastornos o incluso la presencia de cualquier trastorno interiorizado (incluyendo, Ansiedad, Depresión, TOC y TEPT) el punto de corte recomendado es una puntuación de 25 o superior, dado que los valores de sensibilidad, especificidad están en todos los casos por encima de .70 y los índices de Youden son los más altos (.52 o superior).

En resumen, la escala DetectaWeb-Malestar obtiene áreas bajo la curva entre .72 y .93 , por lo que un sujeto tiene una probabilidad entre $72 \%$ y $93 \%$ de presentar el diagnóstico cuando las puntuaciones en la escala superan el punto de corte. Así mismo, presenta una sensibili- 
Tabla 4. Coeficientes alfa de Cronbach basados en la matriz de correlaciones policórica $(\alpha)$ y omega de McDonald

\begin{tabular}{|c|c|c|}
\hline Ítems & $\alpha / \omega$ & Cargas factoriales \\
\hline Factor DM & $.71 / .72$ & \\
\hline 1 & & .67 \\
\hline 2 & & .57 \\
\hline 3 & & .78 \\
\hline Factor D & $.68 / .68$ & \\
\hline 4 & & .66 \\
\hline 5 & & .63 \\
\hline 6 & & .64 \\
\hline Factor $\mathrm{S}$ & $.84 / .85$ & \\
\hline 7 & & .90 \\
\hline 8 & & .86 \\
\hline 9 & & .65 \\
\hline Factor TAS & $.64 / .67$ & \\
\hline 10 & & .65 \\
\hline 11 & & .56 \\
\hline 12 & & .67 \\
\hline Factor FS & $.75 / .67$ & \\
\hline 13 & & .71 \\
\hline 14 & & .83 \\
\hline 15 & & .60 \\
\hline Factor FE & $.64 / .64$ & \\
\hline 16 & & .50 \\
\hline 17 & & .62 \\
\hline 18 & & .71 \\
\hline Factor $\mathrm{Tp} / \mathrm{Ag}$ & $.75 / .75$ & \\
\hline 19 & & .69 \\
\hline 20 & & .77 \\
\hline 21 & & .67 \\
\hline Factor TAG & $.68 / .68$ & \\
\hline 22 & & .54 \\
\hline 23 & & .70 \\
\hline 24 & & .67 \\
\hline Factor TOC & $.55 / .55$ & \\
\hline 25 & & .60 \\
\hline 26 & & .51 \\
\hline 27 & & .51 \\
\hline Factor TEPT & $.69 / .69$ & \\
\hline 28 & & .60 \\
\hline 29 & & .32 \\
\hline 30 & & .74 \\
\hline Total & $.91 / .91$ & \\
\hline
\end{tabular}

Nota . $\mathrm{DM}=$ Depresión mayor; $\mathrm{D}=$ Distimia; $\mathrm{S}=$ Suicidio; $\mathrm{TAS}=$ Trastorno ansiedad por separación; FS = Fobia social; FE = Fobia específica; $\mathrm{Tp} / \mathrm{Ag}=$ Trastorno pánico/agorafobia; $\mathrm{TAG}=$ Trastorno ansiedad generalizada; TOC $=$ Trastorno obsesivo-compulsivo; TEPT $=$ Trastorno por estrés postraumático. dad entre .50 y 1.00 (suicidalidad y trastornos depresivos, respectivamente) y especificidad entre 65 y 97 (trastornos depresivos y $\mathrm{Tp} / \mathrm{Ag}$, respectivamente).

\section{Discusión}

El objetivo principal de este estudio fue examinar la precisión diagnóstica de DetectaWeb-Malestar mediante el análisis de la capacidad del DetectaWeb-Malestar para discriminar entre muestras con diagnóstico de los problemas emocionales: malestar emocional (ansiedad, depresión y suicidalidad) y sin él; Este objetivo se abordó mediante el análisis factorial confirmatorio con muestra clínica y escolar, análisis de fiabilidad, de validez convergente-discriminante y mediante el cálculo de análisis de las curvas ROC, con los consiguiente puntos de corte basados en el mejor equilibrio entre sensibilidad y especificidad de las escalas del cuestionario.

En cuanto los análisis de fiabilidad indican que la escala DetectaWeb-Malestar presenta una alta consistencia interna. Presentando consistencias iguales según el alfa de Cronbach y el coeficiente omega McDonald para la puntuación total $(\alpha / \omega=.91)$, y para las subescalas entre $.55 \mathrm{y}$ .84 según alfa de Cronbach y entre .77 y .91 para Omega. Estos resultados se muestran consistentes con nuestro estudio previo con muestra comunitaria escolar (Piqueras et al., 2018) y con muestra clínica (García-Olcina et al., 2017), además con otros estudios internacionales (van Ballegooijen, Riper, Cuijpers, van Oppen y Smit, 2016).

Como esperábamos, tras poner a prueba los modelos que mejor ajustan obtenidos en el estudio previo con muestra comunitaria y clínica, se confirma la misma estructura factorial, es decir, 10 factores relacionados que evalúan los principales trastornos de ansiedad, depresión y suicidalidad, además el modelo basado en el DSM 5, también presenta buenos ajustes (García-Olcina et al., 2017; Piqueras et al., 2018). El modelo de estructura factorial equivale a otros modelos multidimensionales en los que cada factor corresponde a la dimensión que pretende medir (Chorpita et al., 2005; Chorpita et al., 2000; Orgilés et al., 2012; Sandin et al., 2010).

Respecto a los resultados esperados con respecto a la validez convergente, se aprecian correlaciones positivas y significativas con otras escalas y subescalas de malestar, estos datos son consistentes con las correlaciones de otras pruebas que también evalúan ansiedad y depresión en jóvenes (Cuijpers, Boluijt y Van Straten, 2008; van Ballegooijen et al., 2016). Además, de forma generalizada, se halló que la mayor correlación entre cada subescala de la DetectaWeb-Malestar se dio con aquellas subescalas homólogas de otros cuestionarios, si bien también existieron 
Tabla 5. Correlaciones entre las subescalas y puntuación total del DetectaWeb y otros cuestionarios

\begin{tabular}{|c|c|c|c|c|c|c|c|c|c|c|c|c|c|}
\hline & \multirow[b]{2}{*}{$n$} & \multirow[b]{2}{*}{$\alpha$} & \multicolumn{11}{|c|}{ Escala DetectaWeb-Malestar } \\
\hline & & & $\mathrm{DM}$ & $\mathrm{D}$ & $\mathrm{S}$ & TAS & FS & $\mathrm{FE}$ & $\mathrm{Tp} / \mathrm{Ag}$ & TAG & TOC & TEPT & TOTAL \\
\hline RCADS-30 & 243 & .93 & $.58^{* *}$ & $.57^{* *}$ & $.28^{* *}$ & $.60^{* *}$ & $.66^{* *}$ & $.49^{* *}$ & $.60^{* *}$ & $.60^{* *}$ & $.66^{* *}$ & $.64^{* *}$ & $.85^{* *}$ \\
\hline $\mathrm{DM}$ & & .80 & $.72^{* *}$ & $.71^{* *}$ & $.30^{* *}$ & $.28^{* *}$ & $.52^{* *}$ & $.35^{* *}$ & $.40^{* *}$ & $.41^{* *}$ & $.50^{* *}$ & $.44^{* *}$ & $.70^{* *}$ \\
\hline TP & & .80 & $.50^{* *}$ & $.47^{* *}$ & $.44^{* *}$ & $.40^{* *}$ & $.42^{* *}$ & $.40^{* *}$ & $.60^{* *}$ & $.32^{* *}$ & $.50^{* *}$ & $.61^{* *}$ & $.67^{* *}$ \\
\hline FS & & .83 & $.48^{* *}$ & $.48^{* *}$ & $.20^{* *}$ & $.40^{* *}$ & $.71^{* *}$ & $.37^{* *}$ & $.41^{* *}$ & $.50^{* *}$ & $.45^{* *}$ & $.48^{* *}$ & $.68^{* *}$ \\
\hline TAS & & .79 & $.27^{* *}$ & $.29^{* *}$ & $.14^{*}$ & $.67^{* *}$ & $.41^{* *}$ & $.51^{* *}$ & $.52^{* *}$ & $.30^{* *}$ & $.50^{* *}$ & $.53^{* *}$ & $.63^{* *}$ \\
\hline TAG & & .84 & $.34^{* *}$ & $.33^{* *}$ & .10 & $.53^{* *}$ & $.50^{* *}$ & $.32^{* *}$ & $.41^{* *}$ & $.70^{\star *}$ & $.51^{* *}$ & $.41^{* *}$ & $.64^{* *}$ \\
\hline TOC & & .70 & $.47^{* *}$ & $.42^{* *}$ & $.23^{* *}$ & $.45^{* *}$ & $.47^{* *}$ & $.37^{* *}$ & $.51^{* *}$ & $.44^{* *}$ & $.70^{* *}$ & $.58^{* *}$ & $.70^{* *}$ \\
\hline SCAS & 243 & .64 & $.32^{* *}$ & $.30^{* *}$ & .09 & $.41^{* *}$ & $.33^{* *}$ & $.65^{* *}$ & $.40^{* *}$ & $.27^{* *}$ & $.33^{* *}$ & $.33^{* *}$ & $.52^{* *}$ \\
\hline CRIES & 243 & .93 & $.40^{* *}$ & $.33^{* *}$ & $.22^{* *}$ & $.33^{* *}$ & $.43^{* *}$ & $.28^{* *}$ & $.44^{* *}$ & $.35^{* *}$ & $.34^{* *}$ &. $\mathbf{5 3}^{* *}$ & $.55^{* *}$ \\
\hline Intrusión & & .87 & $.40^{* *}$ & $.32^{* *}$ & $.23^{* *}$ & $.28^{* *}$ & $.40^{* *}$ & $.27^{* *}$ & $.43^{* *}$ & $.35^{* *}$ & $.34^{* *}$ & $.50^{* *}$ & $.53^{* *}$ \\
\hline Evitación & & .88 & $.36^{* *}$ & $.31^{* *}$ & $.18^{* *}$ & $.34^{* *}$ & $.41^{* *}$ & $.27^{* *}$ & $.42^{* *}$ & $.32^{* *}$ & $.31^{* *}$ & $.51^{* *}$ & $.52^{* *}$ \\
\hline SDQ & 49 & .85 & $.50^{* *}$ & $.36^{*}$ & $.40^{* *}$ & .02 & .20 & -.05 & .25 & $.30^{*}$ & $.34^{*}$ & $.37^{* *}$ & $.41^{* *}$ \\
\hline Problemas emocionales & & .70 & $.41^{* *}$ & $.55^{* *}$ & $.42^{* *}$ & $.30^{*}$ & $.34^{*}$ & .20 & $.51^{* *}$ & $.50^{* *}$ & $.54^{* *}$ & $.36^{* *}$ & $.65^{* *}$ \\
\hline Problemas de conducta & & .42 & $.37^{* *}$ & .12 & .06 & .26 & .18 & .05 & .10 & .10 & .27 & $.33^{*}$ & $.30^{*}$ \\
\hline Déficit Atención Hiperactividad & & .70 & $.44^{* *}$ & .16 & .18 & .02 & .09 & -.05 & .06 & .22 & .19 & .23 & .24 \\
\hline Problemas de relación iguales & & .46 & .18 & $.33^{*}$ & .11 & .01 & .26 & -.07 & .19 & .08 & .06 & $.37^{* *}$ & .24 \\
\hline Conducta prosocial & & .53 & .22 & .15 & .21 & $.32^{*}$ & .23 & -.11 & .03 & $.41^{* *}$ & $.31^{*}$ & .07 & $.31^{*}$ \\
\hline MHI-5 & 243 & .74 & $-.53^{* *}$ & $-.44^{* *}$ & $-.35^{* *}$ & $-.14^{*}$ & $-.36^{* *}$ & $-.24^{* *}$ & $-.40^{* *}$ & $-.27^{* *}$ & $-.33^{* *}$ & $-.30^{* *}$ & $-.49^{* *}$ \\
\hline Ansiedad & & & $.65^{* *}$ & $.54^{* *}$ & $.46^{* *}$ & .13 & $.52^{* *}$ & $.30^{*}$ & $.43^{* *}$ & $.48^{* *}$ & $-.33^{* *}$ & $-.30^{* *}$ & $-.49^{* *}$ \\
\hline Depresión & & & $.79^{* *}$ & $.62^{* *}$ & $.55^{* *}$ & .05 & $.56^{* *}$ & .13 & .25 & $.47^{* *}$ & $-.33^{* *}$ & $-.30^{* *}$ & $-.49^{* *}$ \\
\hline WHO-5 & 50 & .86 & $-.62^{* *}$ & $-.37^{* *}$ & $-.58^{* *}$ & .12 & .13 & -.02 & $-.30^{*}$ & -.27 & $-.28^{*}$ & -.16 & $-.33^{*}$ \\
\hline
\end{tabular}

Nota . DM = Depresión mayor; $\mathrm{D}=$ Distimia; $\mathrm{S}=$ Suicidio; TAS = Trastorno ansiedad por separación; FS = Fobia social; FE = Fobia específica; $\mathrm{Tp} / \mathrm{Ag}=$ Trastorno de pánico/agorafobia; $\mathrm{TP}=$ Trastorno de pánico; $\mathrm{TAG}=$ Trastorno ansiedad generalizada; $\mathrm{TOC}=\mathrm{Trastorno}$ obsesivo compulsivo; TEPT = Trastorno estrés post traumático; CRIES-E = Escala de Impacto de Eventos para Niños. Subescala Evitación; CRIES-I = Subescala Intrusión; ${ }^{*} p<.05 ; * *<.01$; en negrita se indican las correlaciones más elevadas.

correlaciones significativas, pero menores con el resto de dimensiones, como sucede entre las subescalas del DetectaWeb-Malestar y del SDQ que evalúan constructos psicopatológicos no relacionadas con los síntomas de malestar emocional (por ejemplo, problemas de comportamiento, hiperactividad, problemas con los compañeros y conducta prosocial). Estos datos también coinciden con los valores hallados correspondientes a otras medidas, como son la correlación de las subescalas de la DASS con el PSWQ (Penn State Worry Questionnaire), que oscila entre .28 y .49 (Zlomke, 2009). En general, los resultados de validez convergente / discriminante de la DetectaWeb-Malestar fueron similares a los encontrados en los estudios previos con muestra clínica (García-Olcina et al., 2017) y en muestra comunitaria (Piqueras et al., 2020).
Referente a las diferencias entre el grupo de procedencia clínica y comunitaria en las escalas DetectaWeb-Malestar, hallamos diferencias significativas en todas las subescalas, excepto en la escala de FS, con magnitudes de las diferencias grandes o próximas a ellas, tal como era de esperar.

Finalmente, se realizó el análisis de curvas ROC para estudiar la sensibilidad y especificidad de la escala DetectaWeb-Malestar. El AUC de la puntuación total de la escala estuvo entre .78 y .80 en función del diagnóstico y los resultados de las puntuaciones de las diferentes subescalas indicaron AUC entre .71 y .93. En general, los valores de alrededor de .70 se pueden considerar valores adecuados de discriminación diagnóstica. Según nuestros datos, el punto de corte óptimo para la escala 
Tabla 6. Diferencias en las puntuaciones de DetectaWeb-Malestar según diagnóstico mediante ADIS-IV-C

\begin{tabular}{|c|c|c|c|c|}
\hline \multirow[b]{2}{*}{ Escala } & $\begin{array}{l}\text { DTW-M } \\
n=202\end{array}$ & $\begin{array}{c}\text { ADIS-IV-C } \\
n=41\end{array}$ & \multirow[t]{2}{*}{$t$} & \multirow[t]{2}{*}{$d$ (Cohen) } \\
\hline & $M(D T)$ & $M(D T)$ & & \\
\hline DM & $1.60 \quad(1.73)$ & $3.30 \quad(2.33)$ & -5.24 & -0.83 \\
\hline D & $1.56(1.60)$ & $3.48 \quad(2.33)$ & -6.49 & -0.96 \\
\hline $\mathrm{S}$ & $0.31 \quad(.96)$ & $0.85 \quad(1.93)$ & -2.71 & -0.35 \\
\hline TAS & $2.70 \quad(1.97)$ & $4.04 \quad(2.47)$ & -3.79 & -0.59 \\
\hline FS & $2.37 \quad(2.07)$ & $4.58 \quad(2.61)$ & -5.94 & -0.94 \\
\hline $\mathrm{FE}$ & $1.57 \quad(1.86)$ & $3.02 \quad(2.53)$ & -4.25 & -0.65 \\
\hline $\mathrm{Tp} / \mathrm{Ag}$ & $1.07 \quad(1.45)$ & $2.87 \quad(2.49)$ & -6.31 & -0.88 \\
\hline TAG & $3.63 \quad(2.38)$ & $5.17 \quad(2.43)$ & -3.77 & -0.64 \\
\hline TOC & 1.15 (1.26) & $2.51 \quad(2.24)$ & -5.39 & -0.74 \\
\hline TEPT & 1.02 & $2.56 \quad(2.49)$ & -5.48 & -0.76 \\
\hline Total & $17.00(10.48)$ & $32.40(15.52)$ & -7.83 & -1.16 \\
\hline
\end{tabular}

Nota . DM = Depresión mayor; D = Distimia; $\mathrm{S}=$ Suicidio; TAS = Trastorno ansiedad por separación; FS = Fobia social; FE = Fobia específica; $\mathrm{Tp} / \mathrm{Ag}=$ Trastorno de pánico/agorafobia; $\mathrm{TAG}=$ Trastorno ansiedad generalizada $; \mathrm{TOC}=$ Trastorno obsesivo compulsivo; $\mathrm{TEPT}=$ Trastorno estrés post traumático. Todas las diferencias resultaron estadísticamente significativas $(p<.001)$.

Tabla 7. Estadísticos descriptivos, Índices de exactitud y AUC de las escalas del DetectaWeb-Malestar para el diagnóstico de trastornos específicos según ADIS-IV-C

\begin{tabular}{|c|c|c|c|c|c|c|c|c|c|c|c|c|c|c|c|}
\hline \multirow{2}{*}{ DTW } & \multicolumn{3}{|c|}{$\mathrm{DM}$} & \multicolumn{3}{|c|}{$\mathrm{D}$} & \multicolumn{3}{|c|}{$\mathrm{S}$} & \multicolumn{3}{|c|}{$\mathrm{Tp} / \mathrm{Ag}$} & \multicolumn{3}{|c|}{ TAG } \\
\hline & Sen & Esp & You & Sen & Esp & You & Sen & Esp & You & Sen & Esp & You & Sen & Esp & You \\
\hline 1 & 1.00 & .34 & .34 & .91 & .30 & .21 & .50 & .86 & .36 & 1.00 & .44 & .44 & 1.00 & .11 & .11 \\
\hline 2 & 1.00 & .56 & .56 & .91 & .58 & .49 & .50 & .91 & .41 & .75 & .63 & .42 & 1.00 & .19 & .19 \\
\hline 3 & 1.00 & .71 & .71 & .64 & .72 & .36 & .50 & .95 & .45 & .50 & .81 & .31 & 1.00 & .36 & .36 \\
\hline 4 & .75 & .82 & .57 & .64 & .84 & .48 & .50 & .99 & .49 & .25 & .89 & .14 & .85 & .47 & .31 \\
\hline 5 & .75 & .93 & .68 & .45 & .92 & .37 & - & - & - & .25 & .93 & .18 & .69 & .66 & .36 \\
\hline 6 & .75 & .96 & .71 & .18 & .95 & .13 & .50 & .99 & .49 & .25 & .96 & .21 & .62 & .77 & .39 \\
\hline 7 & .50 & .98 & .48 & .18 & .99 & .17 & - & - & - & .25 & .97 & .22 & .46 & .84 & .31 \\
\hline 8 & .50 & .99 & .49 & - & - & - & - & - & - & .00 & .98 & -.02 & .39 & .93 & .31 \\
\hline 9 & - & - & - & - & - & - & .50 & 1.00 & .50 & .00 & .99 & -.01 & .15 & .97 & .13 \\
\hline$A U C[I C]$ & \multicolumn{3}{|c|}{$.93[.84-1.00]$} & \multicolumn{3}{|c|}{$.79[.64-.93]$} & \multicolumn{3}{|c|}{$.71[.25-1.00]$} & \multicolumn{3}{|c|}{$.78[.62-.94]$} & \multicolumn{3}{|c|}{$.77[.65-.89]$} \\
\hline$n(\mathrm{c} / \mathrm{nc})$ & \multicolumn{3}{|l|}{$4 / 215$} & \multicolumn{3}{|c|}{$11 / 207$} & \multicolumn{3}{|c|}{$2 / 210$} & \multicolumn{3}{|l|}{$4 / 214$} & \multicolumn{3}{|c|}{$13 / 205$} \\
\hline$M(D T)$ & \multicolumn{3}{|c|}{$1.89(1.87)$} & \multicolumn{3}{|c|}{$1.89(1.94)$} & \multicolumn{3}{|c|}{$0.40(1.19)$} & \multicolumn{3}{|c|}{$1.38(1.80)$} & \multicolumn{3}{|c|}{$3.89(2.45)$} \\
\hline
\end{tabular}

Nota . DTW = DetectaWeb; DM = Depresión mayor; $\mathrm{D}=$ Distimia; $\mathrm{S}=$ Suicidalida, corresponden con las preguntas de la 11(a) a la 11(d) del apartado del trastorno depresivo mayor de la ADIS-IV-C; Tp/Ag = Trastorno de pánico/agorafobia; TAG = Trastorno ansiedad generalizada; Sen = Sensibilidad; Esp = Especificidad; You = Índice de Youden; AUC = Área bajo la curva; IC = Intervalo de confianza al 95\%; $n(\mathrm{c} / \mathrm{nc})=$ número de participantes con trastorno y sin trastorno. En negrita se presenta los valores óptimos para el punto de corte.

total fue de 25. Este punto de corte para la puntuación total podría ser utilizado para detectar e identificar el malestar emocional (cualquier trastorno de ansiedad y depresión) en niños y adolescentes. Sin embargo, el pun- to de corte puede variar según el objetivo. Por ejemplo, si queremos detectar el malestar emocional en una muestra comunitaria, será una prioridad incluir a todos con sintomatología, incluso si esto aumenta la tasa de falsos 
Tabla 7. Continuación...

\begin{tabular}{|c|c|c|c|c|c|c|c|c|c|c|c|c|c|c|c|}
\hline \multirow{2}{*}{ DTW } & \multicolumn{3}{|c|}{ TOC } & \multicolumn{3}{|c|}{ TEPT } & \multicolumn{3}{|c|}{ TAS } & \multicolumn{3}{|c|}{ FS } & \multicolumn{3}{|c|}{ FE } \\
\hline & Sen & Esp & You & Sen & Esp & You & Sen & Esp & You & Sen & Esp & You & Sen & Esp & You \\
\hline 1 & 1.00 & .38 & .38 & .67 & .51 & .18 & .86 & .11 & -.03 & 1.00 & .21 & .21 & .83 & .40 & .23 \\
\hline 2 & 1.00 & .65 & .65 & .67 & .68 & .35 & .86 & .32 & .18 & 1.00 & .37 & .37 & .83 & .58 & .41 \\
\hline 3 & 1.00 & .81 & .81 & .67 & .80 & .47 & .86 & .48 & .34 & .85 & .56 & .40 & .83 & .71 & .54 \\
\hline 4 & .50 & .93 & .43 & .67 & .89 & .56 & .86 & .68 & .54 & .62 & .72 & .33 & .83 & .84 & .68 \\
\hline 5 & .25 & .96 & .21 & .33 & .93 & .26 & .71 & .81 & .52 & .62 & .82 & .44 & .33 & .91 & .24 \\
\hline 6 & .00 & .97 & -.03 & .33 & .98 & .31 & .57 & .89 & .46 & .54 & .88 & .42 & .17 & .94 & .11 \\
\hline 7 & .00 & .98 & -.02 & .33 & .99 & .32 & .57 & .94 & .52 & .46 & .93 & .39 & .17 & .96 & .12 \\
\hline 8 & .00 & .99 & -.01 & - & - & - & .43 & .98 & .41 & .46 & .96 & .42 & .17 & .98 & .14 \\
\hline 9 & - & - & - & .00 & .99 & -.01 & .14 & .99 & .13 & .23 & .99 & .22 & .00 & .99 & -.01 \\
\hline$A U C[I C]$ & \multicolumn{3}{|c|}{$.91[.85-.97]$} & \multicolumn{3}{|c|}{$.72[.33-1.00]$} & \multicolumn{3}{|c|}{$.80[.56-1.00]$} & \multicolumn{3}{|c|}{$.81[.69-.92]$} & \multicolumn{3}{|c|}{$.79[.57-1.00]$} \\
\hline$n(\mathrm{c} / \mathrm{nc})$ & \multicolumn{3}{|c|}{$4 / 214$} & \multicolumn{3}{|c|}{$3 / 215$} & \multicolumn{3}{|c|}{$7 / 211$} & \multicolumn{3}{|c|}{$13 / 205$} & \multicolumn{3}{|c|}{$6 / 212$} \\
\hline$M(D T)$ & \multicolumn{3}{|c|}{$1.38(1.55)$} & \multicolumn{3}{|c|}{$1.28(1.17)$} & \multicolumn{3}{|c|}{$2.93(2.13)$} & \multicolumn{3}{|c|}{$2.74(2.32)$} & \multicolumn{3}{|c|}{$1.82(2.06)$} \\
\hline
\end{tabular}

Nota . DTW $=$ DetectaWeb; TOC $=$ Trastorno obsesivo compulsivo; TEPT $=$ Trastorno estrés post traumático; TAS $=$ Trastorno ansiedad por separación; FS = Fobia social; FE = Fobia específica; Sen = Sensibilidad; Esp = Especificidad; You = Índice de Youden; AUC = Área bajo la curva; IC = Intervalo de confianza al 95\%; $n(\mathrm{c} / \mathrm{nc})=$ número de participantes con trastorno y sin trastorno. En negrita se presenta los valores óptimos para el punto de corte.

positivos. En este caso, una puntuación de corte de 19 o 20 es más sensible que el punto de corte 25 . Por otro lado, si deseamos minimizar el número de falsos positivos y, por tanto, aumentar la especificidad, entonces una puntuación de corte de 27 o incluso 28 sería preferible.

Para detectar la presencia de cualquier trastorno interiorizado (incluyendo, ansiedad, depresión, TOC y TEPT), se encontró que la puntuación de 25 es el punto de corte recomendado, dado que los valores de sensibilidad, especificidad están en todos los casos por encima de .70 y los índices de Youden son los más altos (.52 o superior). Sin embargo, tanto para detectar los trastornos depresivos como los de ansiedad también se puede cambiar de punto de corte, según nuestro objetivo, así para la puntuación de los diferentes trastornos depresivos podemos utilizar el punto de corte 20 para alcanzar una mayor sensibilidad a pesar de que aumente los falsos positivos y el punto de corte de 26 o incluso 27, para una mayor especificidad a riesgo de aumentar los falsos negativos. Referente a las diez subescalas de la escala DetectaWeb-Malestar, los puntos de corte oscilaron entre 2 a 6 , con sensibilidad entre .50 y 1.00 y especificidad entre .56 a .96 , mostrando mejor predicción DM y FE, como ocurre en otro estudio en que DM obtiene mejor predicción (Chorpita et al., 2005), y menor predicción S y TEPT nuestros datos son consistentes con este mismo estudio en el que la DM, FS, TAG y TAS obtienen valores de sensibilidad similares al de nuestro estudio, mien- tras que los valores de especificidad son mayores en nuestro estudio, para el resto de subescalas TOC y Tp/ Ag los valores de sensibilidad son menores mientras que los de especificidad son mayores.

Los resultados del estudio presentan buenas propiedades psicométricas, por lo que la escala DetectaWeb-Malestar es considerada como una prueba de detección del malestar emocional, dado que es un instrumento válido y fiable, con buena sensibilidad y especificidad en comparación con un diagnóstico basado en una entrevista diagnóstica, capaz de detectar sujetos que presenta malestar emocional y detectar aquellos sujetos que están sanos, presentando apoyo empírico sobre su poder de detectar las diferentes subescalas que presenta así como la sintomatología de depresión y ansiedad global.

\section{Conflicto de intereses}

Los autores declaran que no existen conflictos de intereses.

\section{Referencias}

Al-Asadi, A. M., Klein, B. \& Meyer, D. (2015). Multiple comorbidities of 21 psychological disorders and relationships with psychosocial variables: A study of the online assessment and diagnostic system within a web-based population. Journal of Medical Internet Research, 17(2). https://doi.org/10.2196/jmir.4143 
Tabla 8. Índices de exactitud y AUC de la puntuación total del DetectaWeb-Malestar para el diagnóstico de trastornos específicos según ADIS-IV-C

\begin{tabular}{|c|c|c|c|c|c|c|c|c|c|c|c|c|}
\hline \multirow[t]{2}{*}{ DTW } & \multicolumn{3}{|c|}{ ANS } & \multicolumn{3}{|c|}{ DEP } & \multicolumn{3}{|c|}{ EMO } & \multicolumn{3}{|c|}{ INT } \\
\hline & Sen & Esp & You & Sen & Esp & You & Sen & Esp & You & Sen & Esp & You \\
\hline 1 & .97 & .03 & .00 & 1.00 & .03 & .03 & .97 & .03 & .00 & .97 & .03 & .01 \\
\hline 3 & .97 & .07 & .04 & 1.00 & .06 & .06 & .97 & .07 & .04 & .97 & .07 & .05 \\
\hline 5 & .93 & .12 & .06 & 1.00 & .12 & .12 & .94 & .12 & .07 & .95 & .12 & .08 \\
\hline 7 & .93 & .16 & .10 & 1.00 & .15 & .15 & .94 & .17 & .11 & .95 & .17 & .12 \\
\hline 9 & .93 & .20 & .13 & 1.00 & .19 & .19 & .94 & .20 & .15 & .95 & .20 & .16 \\
\hline 10 & .93 & .22 & .16 & 1.00 & .21 & .21 & .94 & .23 & .18 & .95 & .23 & .19 \\
\hline 11 & .90 & .26 & .17 & 1.00 & .25 & .25 & .92 & .26 & .18 & .92 & .27 & .20 \\
\hline 13 & .90 & .33 & .24 & 1.00 & .31 & .31 & .92 & .34 & .26 & .92 & .34 & .27 \\
\hline 15 & .84 & .41 & .26 & .90 & .39 & .30 & .84 & .42 & .26 & .85 & .42 & .28 \\
\hline 17 & .84 & .52 & .37 & .90 & .49 & .40 & .84 & .53 & .37 & .85 & .54 & .40 \\
\hline 18 & .81 & .55 & .37 & .90 & .52 & .43 & .81 & .56 & .38 & .83 & .57 & .41 \\
\hline 19 & .78 & .59 & .37 & .90 & .56 & .46 & .78 & .6 & .38 & .81 & .61 & .42 \\
\hline 20 & .78 & .62 & .41 & .90 & .59 & .50 & .78 & .63 & .42 & .81 & .65 & .46 \\
\hline 21 & .75 & .65 & .41 & .81 & .62 & .43 & .73 & .66 & .4 & .76 & .67 & .43 \\
\hline 22 & .75 & .67 & .42 & .81 & .63 & .45 & .73 & .67 & .41 & .76 & .69 & .45 \\
\hline 23 & .75 & .70 & .45 & .81 & .65 & .47 & .73 & .70 & .44 & .73 & .71 & .45 \\
\hline 24 & .75 & .73 & .49 & .81 & .69 & .50 & .73 & .74 & .47 & .73 & .75 & .48 \\
\hline 25 & .75 & .76 & .52 & .81 & .72 & .53 & .73 & .77 & .51 & .73 & .78 & .52 \\
\hline 26 & .69 & .78 & .47 & .72 & .73 & .46 & .68 & .79 & .47 & .69 & .80 & .49 \\
\hline 27 & .66 & .80 & .46 & .63 & .75 & .39 & .63 & .80 & .43 & .64 & .81 & .45 \\
\hline 28 & .60 & .82 & .43 & .54 & .78 & .32 & .57 & .82 & .40 & .59 & .84 & .43 \\
\hline 29 & .54 & .84 & .38 & .45 & .80 & .25 & .52 & .84 & .37 & .54 & .86 & .40 \\
\hline 30 & .51 & .85 & .37 & .45 & .81 & .27 & .5 & .86 & .36 & .52 & .87 & .39 \\
\hline 31 & .48 & .88 & .36 & .45 & .84 & .29 & .47 & .88 & .36 & .50 & .90 & .40 \\
\hline 32 & .48 & .88 & .37 & .45 & .84 & .30 & .47 & .89 & .36 & .50 & .90 & .40 \\
\hline 33 & .45 & .90 & .35 & .45 & .87 & .32 & .44 & .91 & .36 & .45 & .92 & .37 \\
\hline 34 & .45 & .91 & .36 & .45 & .87 & .33 & .44 & .91 & .36 & .45 & .92 & .37 \\
\hline 35 & .45 & .91 & .37 & .45 & .88 & .33 & .44 & .92 & .37 & .45 & .93 & .38 \\
\hline 37 & .42 & .94 & .37 & .36 & .90 & .27 & .39 & .95 & .34 & .38 & .95 & .33 \\
\hline 39 & .39 & .96 & .36 & .36 & .93 & .29 & .36 & .97 & .33 & .35 & .97 & .33 \\
\hline 41 & .33 & .97 & .30 & .27 & .94 & .21 & .31 & .97 & .29 & .31 & .98 & .29 \\
\hline 43 & .27 & .97 & .24 & .27 & .95 & .22 & .26 & .98 & .24 & .26 & .98 & .24 \\
\hline 45 & .24 & .98 & .22 & .27 & .96 & .23 & .23 & .98 & .22 & .23 & .99 & .22 \\
\hline 47 & .21 & .98 & .19 & .18 & .96 & .14 & .18 & .98 & .17 & .19 & .99 & .18 \\
\hline 49 & .15 & .98 & .13 & .18 & .97 & .15 & .13 & .98 & .11 & .14 & .99 & .13 \\
\hline 50 & .15 & .99 & .14 & .18 & .97 & .16 & .13 & .99 & .12 & .14 & .99 & .13 \\
\hline 56 & .12 & .99 & .11 & .09 & .97 & .06 & .10 & .99 & .09 & .11 & .99 & .11 \\
\hline 58 & .03 & .99 & .02 & .00 & .99 & -.01 & .02 & .99 & .02 & .04 & 1.00 & .04 \\
\hline 65 & .03 & 1.00 & .03 & .00 & .99 & -.01 & .02 & 1.00 & .02 & .02 & 1.00 & .02 \\
\hline$A U C[I C]$ & \multicolumn{3}{|c|}{$.78[.69-.88]$} & \multicolumn{3}{|c|}{$.80[.69-.91]$} & \multicolumn{3}{|c|}{$.78[.70-.87]$} & \multicolumn{3}{|c|}{$.80[.72-.88]$} \\
\hline$n(\mathrm{c} / \mathrm{nc})$ & \multicolumn{3}{|c|}{$33 / 210$} & \multicolumn{3}{|c|}{$11 / 207$} & \multicolumn{3}{|c|}{$38 / 205$} & \multicolumn{3}{|c|}{$42 / 201$} \\
\hline
\end{tabular}

Nota . DTW = DetectaWeb; ANS = Trastornos de ansiedad; DEP = Trastornos depresivos; Trastornos emocionales; EMO = Trastornos emocionales; Trastornos interiorizados. Sen = sensibilidad; Esp = Especificidad; You = Índice de Youden; AUC = Área bajo la curva; IC $=$ Intervalo de confianza al $95 \% ; n(\mathrm{c} / \mathrm{nc})=$ número de participantes con trastorno y sin trastorno. En negrita se presenta los valores óptimos para el punto de corte. 
Alcántara-Jiménez, M. M., y Garcia-Lopez, L. (2017). Revisión de los procedimientos de evaluación observacional y cognitiva en población con ansiedad social. Revista de Psicopatología y Psicología Clínica, 22(3), 243-260. https://doi.org/10.5944/ rppc.vol.22.num.3.2017.19187.

American Psychiatric Association. (2013). Diagnostic and Statistical Manual of Mental Disorders. Washington DC: APA. https://doi.org/10.1176/appi. books. 9780890425596.744053

Bentler, P. M. (1990). Comparative fit indexes in structural models. Psychological Bulletin, 107(2), 238-246. https://doi. org/10.1037/0033-2909.107.2.238

Berwick, D. M., Murphy, J. M., Goldman, P. A., Ware, J. E., Barsky, A. J. \& Weinstein, M. C. (1991). Performance of a five-item mental health screening test. Medical Care, 29(2), 169-176. https://doi.org/10.1097/00005650-199102000-00008

Boyce, W., Torsheim, T., Currie, C. \& Zambon, A. (2006). The family affluence scale as a measure of national wealth: Validation of an adolescent self-report measure. Social Indicators Research, 78(3), 473-487. https://doi.org/10.1007/ s11205-005-1607-6

Brown, T. A., Di Nardo, P. \& Barlow, D. H. (1994). Anxiety disorders interview schedule adult version (ADIS-IV): Client interview schedule. San Antonio, TX: Psychological Corporation.

Browne, M. W. \& Cudeck, R. (1993). Alternative ways of assessing model fit. In J. S. (Eds. . Bollen, K. A. \& Long (Ed.), Testing Structural Equation Models (Vol. 154, pp. 136-162). Newbury Park, CA: Sage Publications.

Chau, P. \&. K. (1997). Reexamining a model for evaluating information center success using a structural equation modeling approach. Decision Sciences, 28(2), 309-334. https://doi.org/10.1111/j.1540-5915.1997.tb01313.x

Chorpita, B. F., Moffitt, C. E. \& Gray, J. (2005). Psychometric properties of the Revised Child Anxiety and Depression Scale in a clinical sample. Behaviour Research and Therapy, 43(3), 309-322. https://doi.org/10.1016/j.brat.2004.02.004

Chorpita, B. F., Yim, L., Moffitt, C. E., Umemoto, L. A. \& Francis, S. E. (2000). Assessment of symptoms of DSM-IV anxiety and depression in children: A revised child anxiety and depression scale. Behaviour Research and Therapy, 38, 835855. https://doi.org/10.1016/s0005-7967(99)00130-8

Cohen, J. (1988). Statistical power analysis for the behavioral sciences. Hillsdale, NJ: Lawrence Erlbaum Associates Publishers.

Cuijpers, P., Boluijt, P. \& Van Straten, A. (2008). Screening of depression in adolescents through the Internet: Sensitivity and specificity of two screening questionnaires. European Child and Adolescent Psychiatry, 17(1), 32-38. https://doi. org/10.1007/s00787-007-0631-2

Cummings, C. M., Caporino, N. E. \& Kendall, P. C. (2014). Comorbidity of anxiety and depression in children and adolescents: 20 years after. Psychological Bulletin, 140(3), 816-845. https://doi.org/10.1037/a0034733

Currie, C. E., Elton, R. A., Todd, J. \& Platt, S. (1997). Indicators of socioeconomic status for adolescents: the WHO Health Behaviour in school-aged children survey. Health Education Research, 12(3), 385-397. https://doi.org/10.1093/ her/12.3.385 de Ross, R., Gullone, E. \& Chorpita, B. F. (2002). The Revised Child Anxiety and Depression Scale: A psychometric investigation with Australian youth. Behaviour Change, 19(2), 90-101. https://doi.org/10.1375/bech.19.2.90.

Dunn, T. J., Baguley, T. \& Brunsden, V. (2014). From alpha to omega: A practical solution to the pervasive problem of internal consistency estimation. British Journal of Psychology, 105(3), 399-412. https://doi.org/10.1111/bjop.12046

Ebesutani, C., Bernstein, A., Nakamura, B. J., Chorpita, B. F., Higa-Mcmillan, C. K. \& Weisz, J. R. (2010). Concurrent validity of the child behavior checklist DSM-Oriented Scales: Correspondence with DSM diagnoses and comparison to syndrome scales. Journal of Psychopathology and Behavioral Assessment, 32(3), 373-384. https://doi.org/10.1007/s10862009-9174-9

Ebesutani, C., Drescher, C. F., Reise, S. P., Heiden, L., Hight, T., Damon, J. D. \& Young, J. (2012). The Loneliness QuestionnaireShort Version: An evaluation of reverse-worded and nonreverse-worded items via item response theory. Journal of Personality Assessment, 94(4), 427-437. https://doi.org/10.10 80/00223891.2012.662188

García-Olcina, M. G., Piqueras, J.A. y Martínez-González, A. E.(2014). Datos preliminares de la validación del cuestionario de detección vía web para los trastornos emocionales (DETECTA-WEB) en adolescentes españoles. Revista de Psicología Clínica Con Niños y Adolescentes, 1(1), 69-77.

García-Olcina, M., Rivera-Riquelme, M., Cantó-Diez, T. J., TomáBerenguer, M. R., Bustamante, R. y Piqueras, J. A. (2017). Detección online de trastornos emocionales en población clínica de niños y adolescentes: Escala DetectaWeb-Malestar. Revista de Psicología Clínica Con Niños y Adolescentes, 4(3), $35-45$.

García-Villamisar, D., Vidal, A. y Yenes, E. (2002). Estructura de los síntomas de la ansiedad en la infancia. Un estudio exploratorio a través de la Multidimensional Anxiety Scale for Children-Spanish Version (MASC-SV). Psiquis: Revista de Psiquiatría, Psicología y Psicosomática, 23(2), 5-18.

Goodman, R. (1997). The Strengths and Difficulties Questionnaire: A research note. Journal of Child Psychology and Psychiatry, 38(5), 581-6. https://doi.org/10.1111/j.1469-7610.1997.tb01545.x

Hernández Guzmán, L., Bermúdez Ornelas, G., Spence, S., González Montesinos, M. J., Martínez Guerrero, J., Aguilar Villalobos, J. y Gallegos Guajardo, J. (2010). Versión en español de la Escala de Ansiedad para Niños de Spence (SCAS). Revista Latinoamericana de Psicología, 42(1), 13-24.

Jöreskog, K. G. \& Sörbom, D. (1993). LISREL 8: Structural equation modeling with the SIMPLIS command language. Hillsdale, NJ: Lawrence Erlbaum Associates, Inc.

Kovacs, M. (1992). Children's Depression Inventory CDI Manual. New York: Multi-Health Systems., 1-800.

Lipsey, M, Wilson, D. (2001). Practical Meta-analysis. Thousand Oaks, CA: Sage Publications.

March, J. S., Parker, J. D., Sullivan, K., Stallings, P. \& Conners, C. K. (1997). The Multidimensional Anxiety Scale for Children (MASC): Factor structure, reliability, and validity. Journal of the American Academy of Child and Adolescent Psychiatry, 36(4), 554-565. https://doi.org/10.1097/00004583-199704000-00019

McDonald, R. P. (1999). Test Theory A Unified Treatment. Mahwah,NJ:Erlbaum.https://doi.org/10.4324/9781410601087 
Melton, T. H., Croarkin, P. E., Strawn, J. R. \& McClintock, S. M. (2016). Comorbid anxiety and depressive symptoms in children and adolescents: A Systematic review and analysis. Journal of Psychiatric Practice, 22(2), 84-98. https://doi. org/10.1097/PRA.0000000000000132

Metz, C. E. (1978). Basic principles of ROC analysis. Seminars in Nuclear Medicine, 8(4), 283-298. https://doi.org/10.1016/ S0001-2998(78)80014-2

Moran, V. E., Cupani, M., Azpilicueta, A. E., Piqueras, J. A., \& Garcia Lopez, L. J. (2019). Rasch model analysis of the Brief Version of the Social Phobia and Anxiety Inventory (SPAI-B) in Argentinean and Spanish samples. Revista de Psicopatología y Psicología Clínica, 23(2), 211-220. doi: 10.5944/rppc. vol.23.num.3.2018.22516

Muris, P., Simon, E., Lijphart, H., Bos, A., Hale, W., Schmeitz, K., ... Wolters, L. (2017). The Youth Anxiety Measure for DSM-5 (YAM-5): Development and First Psychometric Evidence of a New Scale for Assessing Anxiety Disorders Symptoms of Children and Adolescents. Child Psychiatry and Human Development, 48(1). https://doi.org/10.1007/s10578-016-0648-1

Orgilés, M., Fernández-Martínez, I., Guillén-Riquelme, A., Espada, J. P. \& Essau, C. A. (2016). A systematic review of the factor structure and reliability of the Spence Children's Anxiety Scale. Journal of Affective Disorders, 190, 333-340. https://doi.org/10.1016/j.jad.2015.09.055

Orgilés, M., Méndez, X., Spence, S. H., Huedo-Medina, T. B. \& Espada, J. P. (2012). Spanish validation of the spence children's anxiety scale. Child Psychiatry and Human Development, 43(2), 271-281. https://doi.org/10.1007/s10578-011-0265-y

Ortuño-Sierra, J., Fonseca-Pedrero, E., Paino, M., Sastre I Riba, S. \& Muñiz, J. (2015). Screening mental health problems during adolescence: Psychometric properties of the Spanish version of the strengths and difficulties Questionnaire. Journal of Adolescence, 38, 49-56. https://doi.org/10.1016/j.adolescence.2014.11.001

Perrin, S., Meiser-Stedman, R. \& Smith, P. (2005). The Children's Revised Impact of Event Scale (CRIES): Validity as a Screening Instrument for PTSD. Behavioural and Cognitive Psychotherapy, 33(4), 487. https://doi.org/10.1017/S1352465805002419

Piqueras, J. A., García-Olcina, M., Rivera-Riquelme, M., Martínez-González, A. E. \& Cuijpers, P. (2020). DetectaWebDistress Scale: a global and multidimensional Web-Based Screener for emotional disorders symptoms in Children and Adolescents. Frontiers in Psychology.

Piqueras, J. A., Garcia-Olcina, M., Rivera-Riquelme, M., Rodriguez-Jimenez, T., Martinez-Gonzalez, A. E. \& Cuijpers, P. (2017). DetectaWeb Project: Study protocol of a web-based detection of mental health of children and adolescents. BMJ Open, 7(10), e017218.

Piqueras, J. A., Martín-Vivar, M., Sandin, B., San Luis, C. \& Pineda-Sanchez, D. (2017). The Revised Child Anxiety and Depression Scale: A systematic review and reliability generalization meta-analysis. Journal of Affective Disorders. https://doi.org/10.1016/j.jad.2017.04.022

Piqueras, J. A., Pineda, D., Martin-Vivar, M. \& Sandín, B. (2017). Confirmatory factor analysis and psychometric properties of the Revised Child Anxiety and Depression Scale-30 (RCADS-30) in clinical and non-clinical samples. Revista de Psicopatología y Psicología Clínica, 22(3), 183-196. https:// doi.org/10.5944/rppc.vol.22.num.3.2017.19332
Pineda, D., Martín-Vivar, M., Sandín, B. \& Piqueras, J. A. (2018). Factorial invariance and norms of the 30-item shortenedversion of the Revised Child Anxiety and Depression Scale (RCADS-30). Psicothema, 30(2), 232-237. https://doi. org/10.7334/psicothema2017.276

Reynolds, C. R. \& Richmond, B. O. (1997). What I think and feel: A revised measure of children's manifest anxiety. Journal of Abnormal Child Psychology, 25(1), 15-20. https://doi.org/10. 1007/bf00919131

Sánchez-Teruel, D., Muela-Martínez, J. A., \& García-León, A. (2018). Variables de riesgo y protección relacionadas con la tentativa de suicidio. Revista de Psicopatología y Psicología Clínica, 23(3), 221-229. https://doi.org/10.5944/rppc.vol.23. num.3.2018.19106

Sandín, B., Chorot, P., Valiente, R. M., \& Chorpita, B. F. (2010). Development of a 30-item version of the Revised Child Anxiety and Depression Scale. Revista de Psicopatología y Psicología Clínica, 15(3), 165-178.

Sandín, B., Valiente, R. M. y Chorot, P. (2009). RCADS : evaluación de los síntomas de los trastornos de ansiedad y depresión en niños y adolecentes. Revista de Psicopatología y Psicología Clínica, 14(3), 193-206. https://doi.org/http:// dx.doi.org/10.5944/rppc.vol.14.num.3.2009.4078

Schumacker, R. E. \& Lomax, R. G. (2004). A beginner's guide to structural equation modeling. New York: Routledge Taylor \& Francis Group. https://doi.org/10.1080/10705510802154356

Silverman, W. K., Albano, A. M. y Sandín, B. (2003). ADIS-IV/C: Entrevista para el diagnóstico de los trastornos de ansiedad en niños según el DSM-IV. Entrevista para el niño. Madrid: Klinik.

Spence, S. H. (1997). Structure of anxiety symptoms among children: A confirmatory factor-analytic study. Journal of Abnormal Psychology, 106(2), 280-297. https://doi.org/10. 1037/0021-843X.106.2.280

van Ballegooijen, W., Riper, H., Cuijpers, P., van Oppen, P. \& Smit, J. H. (2016). Validation of online psychometric instruments for common mental health disorders: A systematic review. BMC Psychiatry, 16(1), 45. https://doi.org/10.1186/ s12888-016-0735-7

Veit, C. T. \& Ware, J. E. (1983). The Structure of Psychological Distress and Well-Being in General Populations. Journal of Consulting and Clinical Psychology, 51(5), 730-742.

Walter, J. G., Kahn, S. A., Noe, J. D., Schurman, J. V, Miller, S. A. \& Greenley, R. N. (2016). Feeling fine: Anxiety and depressive symptoms in youth with established IBD. Inflammatory Bowel Diseases, 22(2), 402-408. https://doi.org/10.1097/MIB. 0000000000000657

World Health Organization (WHO) (1998). Well-being measures in primary health care: the DepCare project. Copenhagen, Denmark: WHO Reg Off Eur, 1998:b32.

World Health Organization. (2014). Health for the world's adolescents. A second chance in the second decade. Geneva: WHO.

Youden, W. J. (1950). Index for rating diagnostic tests. Cancer, 3(1), 32-35. https://doi.org/10.1002/1097-0142(1950)3:1<32::AIDCNCR2820030106>3.0.CO;2-3

Zlomke, K. R. (2009). Psychometric properties of internet administered versions of the Penn State Worry Questionnaire (PSWQ) and Depression, Anxiety, and Stress Scale (DASS). Computers in Human Behavior, 25(4), 841-843. https://doi. org/10.1016/j.chb.2008.06.003 